Article

\title{
Reduction of Salmonella Typhimurium Cecal Colonisation and Improvement of Intestinal Health in Broilers Supplemented with Fermented Defatted 'Alperujo', an Olive Oil By-Product
}

\author{
Agustín Rebollada-Merino ${ }^{1,2} \mathbb{D}^{\circ}$, María Ugarte-Ruiz ${ }^{1, *} \mathbb{*}$, Marta Hernández ${ }^{3,4}$, \\ Pedro Miguela-Villoldo 1,5, David Abad ${ }^{3}$ (D), David Rodríguez-Lázaro ${ }^{3,4}\left(\mathbb{D}\right.$, Lucía de Juan ${ }^{1,5}$, \\ Lucas Domínguez $^{1,5}$ and Antonio Rodríguez-Bertos ${ }^{1,2}$ \\ 1 VISAVET Health Surveillance Centre, Complutense University of Madrid, 28040 Madrid, Spain; \\ agusrebo@ucm.es (A.R.-M.); pedromig@ucm.es (P.M.-V.); dejuan@visavet.ucm.es (L.d.J.); \\ lucasdo@visavet.ucm.es (L.D.); arbertos@ucm.es (A.R.-B.) \\ 2 Department of Internal Medicine and Animal Surgery, Faculty of Veterinary Medicine, \\ Complutense University of Madrid, 28040 Madrid, Spain \\ 3 Laboratorio de Biología Molecular y Microbiología, Instituto Tecnológico Agrario de Castilla y León, \\ 47071 Valladolid, Spain; ita-HerPerMa@itacyl.es (M.H.); abagarda@itacyl.es (D.A.); \\ drlazaro@ubu.es (D.R.-L.) \\ 4 Área de Microbiología, Departamento de Biotecnología y Ciencia de los Alimentos, Universidad de Burgos, \\ 09001 Burgos, Spain \\ 5 Department of Animal Health, Faculty of Veterinary Medicine, Complutense University of Madrid, \\ 28040 Madrid, Spain \\ * Correspondence: maria.ugarte@ucm.es; Tel.: +34-913-944-097
}

Received: 28 September 2020; Accepted: 20 October 2020; Published: 21 October 2020

Simple Summary: Salmonella spp. is a bacterium that places human health at risk by consuming eggs and poultry. In the European Union, the use of antimicrobials to treat salmonellosis in aviculture is no longer permitted due to the resistance to treatment of some bacteria, such as Salmonella spp. For this reason, compounds derived from natural food sources are being increasingly tested to assess their efficacy against Salmonella spp. In this study, chickens were given dietary supplements in the form of fermented defatted 'alperujo', a modified olive oil by-product, after which they were infected with Salmonella Typhimurium. The chickens given the supplement showed a healthy gut and a reduction in the amount of Salmonella spp. in the cecum. In conclusion, this olive oil by-product may contribute to preventing and controlling salmonellosis in farms, as well as reducing environmental contamination.

Abstract: Salmonella spp. contaminates egg and poultry meat leading to foodborne infections in humans. The emergence of antimicrobial-resistant strains has limited the use of antimicrobials. We aimed to determine the effects of the food supplement, fermented defatted 'alperujo' (FDA), a modified olive oil by-product, on Salmonella Typhimurium colonisation in broilers. One hundred and twenty 1-day-old broilers were divided into four experimental groups-two control groups and two treated groups, and challenged with $S$. Typhimurium at day 7 or 21 . On days $7,14,21,28$, 35 , and 42 of life, duodenum and cecum tissue samples were collected for histopathological and histomorphometric studies. Additionally, cecum content was collected for Salmonella spp. detection by culture and $\mathrm{qPCR}$, and for metagenomic analysis. Our results showed a significant reduction of Salmonella spp. in the cecum of 42-day-old broilers, suggesting that fermented defatted 'alperujo' limits Salmonella Typhimurium colonization in that cecum and may contribute to diminishing the risk of carcass contamination at the time of slaughter. The improvement of the mucosal integrity, observed histologically and morphometrically, may contribute to enhancing intestinal health and to limiting Salmonella spp. colonisation in the host, mitigating production losses. These results could 
provide evidence that FDA would contribute to prophylactic and therapeutic measures to reduce salmonellosis prevalence in poultry farms.

Keywords: antimicrobial alternatives; fermented defatted 'alperujo'; intestinal health; olive oil by-products; Salmonella Typhimurium

\section{Introduction}

Salmonella spp. is Gram-negative intracellular enteric bacterium of public health concern. It is responsible for over 90,000 cases of zoonotic food-borne infections per year in the European Union (EU), according to the last European Food and Safety Authority (EFSA) report [1]. In recent years, different serovars were considered responsible for disease outbreaks, and Salmonella Typhimurium and $S$. Enteritidis were the most prevalent in the European Union [1].

The main source of human infections in high-income countries is associated with the consumption of eggs and poultry meat, which usually become contaminated during the slaughtering process through the food chain [1]. Salmonellosis in poultry causes decreased growth and eventual mortality in chickens [2], although normally chickens are asymptomatic carriers and shed the bacteria in their feces [3,4]. Due to the role of poultry in disseminating Salmonella spp., control measures, such as those included in National Control Plans, have been implemented in the EU to reduce the prevalence of salmonellosis and other foodborne diseases in poultry production [5].

The emergence of antimicrobial-resistant strains limits the use of antimicrobials to treat Salmonella spp. infections in poultry in the EU $[2,3,6]$. For this reason, the complementary use of compounds with antimicrobial proprieties as feed additives or supplements has been promoted [3]. These supplements include probiotic, prebiotic, phytobiotic, and nutraceutical products $[7,8]$.

During the olive oil two-phase extraction system, a semisolid by-product known as two-phase mill waste, olive pomace, or 'alperujo' is obtained [9]. The olives contain phenolic compounds that are retained in olive oil and in the by-products generated during olive oil processing [10], like olive pomace [11]. In vitro, olive oil by-products stimulate the synthesis of metabolites with bactericidal proprieties, and immune response [7,8,12]. In particular, oleuropein, decarboxymethyl ligstroside tyroxol, and hydroxytyrosol are known to possess antimicrobial proprieties $[8,10,12,13]$, which could help to limit pathogen bacterial infection, colonisation, and excretion. Olive oil extracts were said to inhibit the growth of Salmonella Typhimurium and S. Enteritidis in vitro $[8,10,12,13]$. Besides, in broilers, olive pomace extract has demonstrated anti-inflammatory proprieties [11]. However, mass application of these extracts in animal production is difficult and costly.

In broilers and laying hens, direct supplementation of fermented defatted 'alperujo' (FDA) has been shown to improve intestinal morphology and induce changes in the microbiota [14,15], suggesting a beneficial effect on intestinal health. The improvement in the response of intestinal mucosa to eventual damage may lead to controlling Salmonella spp. colonisation in the gut and thus limiting transmission within the farm and through the food chain [16]. However, the effects of FDA supplementation on controlling pathogenic bacteria as Salmonella spp. in the poultry gut has not yet been investigated.

The aim of this study was to evaluate the effects that FDA has on the intestinal mucosal morphology (duodenum and cecum) and on cecal reduction of Salmonella Typhimurium in broilers infected with Salmonella Typhimurium at 7 or 21 days old. We assumed that the antimicrobial proprieties previously described in olive oil by-products could contribute to mitigating Salmonella spp. infection in broilers. 


\section{Materials and Methods}

\subsection{Ethical Approval and Animal Welfare}

All the experimental procedures were approved by the Animal Care and Ethics Committee of the Complutense University of Madrid in compliance with the Community of Madrid (PROEX 152/19). Animal experiments took place in the biosafety level 3 (BSL-3) facilities of the VISAVET Health Surveillance Centre. One-day-old male Ross 308 broilers were housed according to the European legislation on animal welfare (Directive 2010/63/EU): water and food were provided ad libitum, and temperature and light/dark cycles controlled according to age.

\subsection{Animal Groups and Feed}

The distribution of the experiment and the animals included two separate boxes with two cages each-one treated group and one control group per box, with 30 animals per cage ( $n=120$ animals). Controls were fed with conventional broiler feed. Since their arrival, treated animals received the same commercial feed as the control group but were given a supplement of $2 \%$ FDA as previously described [14,15]. FDA composition is detailed in Table 1 [14].

Table 1. Fermented defatted "alperujo" (FDA) composition.

\begin{tabular}{cc}
\hline Determination & Results \\
\hline Moisture $103^{\circ}$ (\%w.w.) & 12.20 \\
Crude protein (Kjeldahl) (\%w.w.) & 6.40 \\
Brute fat (\%w.w.) & 3.00 \\
Ash content (\%w.w.) & 7.70 \\
Lignin (\%w.w.) & 23.30 \\
Acid detergent fibre (\%w.w.) & 39.20 \\
Neutral detergent fibre (\%w.w.) & 49.30 \\
Tannins (\%w.w.) & 0.06 \\
Oleic acidity index (\%w.w.) & 46.10 \\
Peroxide value (\%w.w.) & 7.90 \\
Total polyphenols (meq/kg) & 0.89 \\
Crude fibre (\%w.w.) & 27.70 \\
\hline
\end{tabular}

\subsection{Salmonella Typhimurium Challenge}

Immediately after arrival, animals were tested for Salmonella spp. following ISO 6579-1:2017 [17], obtaining negative results. After $3 \mathrm{~h}$ of water restriction, at day 7 or 21 , all animals in boxes 1 or 2 , respectively, were challenged with $3.3 \times 10^{5} \mathrm{CFU} / \mathrm{mL}$ of monophasic variant of colistin-resistant $S$. Typhimurium (mcr-1 positive) suspended in a total volume of $330 \mathrm{~mL}$ of drinking water. A clinical examination was performed twice-a-day upon arrival and at the end of the experiment, with special focus on water and food consumption, animal welfare, emergence of clinical signs after the Salmonella Typhimurium challenge, and eventual mortality.

\subsection{Postmortem Examination and Samplings}

Five randomly-selected animals from each group $(n=20)$ were sedated with diazepam $(1 \mathrm{mg} / \mathrm{kg}$ intramuscular) and euthanised with an overdose of sodium pentobarbital (100 mg/kg intravenous) on days 7, 14, 21, 28, 35, and 42 of life. A complete post-mortem survey was performed for each animal. Cecum feces were collected in parallel for Salmonella spp. detection using traditional culture and conserved at $-80{ }^{\circ} \mathrm{C}$ for molecular analysis and further metagenomic studies. Duodenum and cecum tissue samples were fixed in $10 \%$ of commercial buffered formaldehyde solution. 


\subsection{Salmonella spp. Detection and Culture Conditions}

Salmonella spp. detection was performed following a protocol already published [18], although samples were streaked onto SMID2 agar plates (BioMérieux, Marcy-l'Étoile, France) instead of ColR agar plates (BioMérieux), using serial dilutions in order to quantify the presence of this Salmonella spp. The agar plates were examined after incubation at $37^{\circ} \mathrm{C}$ for $24 \mathrm{~h}$ under aerobic conditions. All colonies resembling Salmonella spp., according to the manufacturer's instructions, were counted.

\subsection{Real-Time PCR for Salmonella spp. Detection}

The detection of Salmonella spp. by real-time PCR (qPCR) was based on previously-described assays [19,20], using $3 \mu \mathrm{L}$ of the same total cecal DNA extracted for 16S rRNA Library Preparation and sequencing as a template. Reactions were run on an Applied BioSystems 7500 (Applied BioSystems, Foster City, CA, USA). Amplification was performed using an initial hot-start step at $95^{\circ} \mathrm{C}$ for $10 \mathrm{~min}$, followed by 40 cycles of a denaturation step at $95^{\circ} \mathrm{C}$ for $30 \mathrm{~s}$ and an annealing/extension step at $55^{\circ} \mathrm{C}$ for $60 \mathrm{~s}$. Fluorescence was only recorded at the end of the annealing/extension step. Three qPCR replicates were used for each sample.

\subsection{S rRNA Library Preparation and Sequencing}

Total DNA was extracted from $220 \mathrm{mg}$ of cecal content using a commercial kit (QIAamp DNA Stool Mini Kit, Qiagen, Hilden, Germany) and DNA concentration was determined using a fluorometer (Qubit fluorometer, Invitrogen, Carlsbad, CA, USA). Microbial diversity was assessed by analyzing sequences of the V3-V4 region of the 16S rRNA gene. The primers and PCR conditions used for this analysis were as previously reported [21]. Sample multiplexing, library purification, and sequencing were performed as described in the "16S Metagenomic Sequencing Library Preparation" guide by Illumina (San Diego, CA, USA). Libraries were sequenced on an Illumina MiSeq platform that provided 300-bp paired-end reads.

\subsection{Bioinformatics and Data Analysis}

Raw demultiplexed sequence data was processed using QiimeReporter (https:/github.com/ dabadgarcia/qiimereporter, Instituto Tecnologico Agrario de Castilla y Leon, Valladolid, Spain). This straightforward pipeline for the analysis of amplicon sequences integrates basic Qiime2 commands [22] with the R programming language. In brief, the DADA2 package [23] was used to filter reads, merge paired ends, remove chimeras, and assign amplicon sequence variants (ASV). Then, a pre-trained Naïve Bayes classifier [24] was used to obtain the taxonomic assignment of the ASVs, using SILVA database version 132 (SILVA ribosomal RNA database project, Bremen, Germany) as a reference [25], which resulted in a table containing the microbial composition for each of the samples. Raw reads are available in the BioProject database with ID PRJNA643396, samples SAMN16203983 to SAMN16203863 (http://www.ncbi.nlm.nih.gov/bioproject/).

\subsection{Histological Processing, and Histopathological and Histomorphometric Analysis}

After fixation, routine histological processing and hematoxylin-eosin staining was carried out as described elsewhere [14,15]. A histopathological study focused on morphological features (villous stunting in duodenum, epithelial injury, crypt hyperplasia, crypt distortion, lamina propria oedema, lacteal dilation, mucosal fibrosis, and hemorrhages) and inflammation (intraepithelial lymphocytes, lamina propria lymphocytes, heterophils, eosinophilic granular cells, and/or macrophages, and mucosal-associated lymphoid tissue) was performed at 7 and 14 days post-infection (dpi). In addition, samples of duodenum and cecum were subjected to a histomorphometric analysis at $400 \times$ magnifications employing an image analyzer (Leica Application Suite, Leica, Wetzlar, Germany), as described elsewhere [14,15]. Twenty intact and well-oriented villi in duodenum and 20 crypts in duodenum and cecum were measured in each animal. Duodenum villi were measured from the top to the crypt-villus 
junction. Crypts were measured from the crypt-villus junction in the duodenum or the mucosal surface in the cecum to the basement membrane.

\subsection{Statistical Analysis}

A statistical analysis of Salmonella spp. culture, qPCR, and histomorphometry was performed using Mann-Whitney and Fisher exact tests following IBM SPSS Statistics Software v25 (IBM, Armonk, NY, USA). The level for statistical significance was set at $p<0.05$.

\section{Results}

\subsection{Clinical Signs and Gross Findings}

During the experiment, no clinical signs or mortality were reported. The post-mortem examination revealed mild diffuse chronic catarrhal enteritis in animals belonging to the control group. Occasional hepatic congestion or steatosis, not associated with treatment or challenge age, was also reported.

\subsection{Salmonella Typhimurium Colonisation in the Cecum}

In 7-day-old challenged chickens, at 7 dpi (14 days old), the load of Salmonella spp. in the cecum was significantly lower than controls by culture $(p=0.008)$, and almost by qPCR $(p=0.056)$. At 14 dpi (21 days old), there were significant differences in the Salmonella spp. cecal load between the control and treated group by qPCR $(p=0.032)$ but not by culture $(p>0.05)$. At 21,28 , and $35 \mathrm{dpi}(28,35$, and 42 days old, respectively) there were no significant differences in the cecal Salmonella spp. load among groups by culture $(p>0.05)$ or by qPCR $(p>0.05)$. (Figures 1 and 2 , Table 2$)$.

Table 2. Results of culture (CFU/g) and qPCR (genome equivalent/g) in 7-day-old challenged chickens.

\begin{tabular}{ccccc}
\hline Days-Old & & Control & Treated & $p$-Value ${ }^{\mathbf{1}}$ \\
\hline 7 & Culture & $<10^{2}$ & $<10^{2}$ & 1.000 \\
& qPCR & $*$ & $*$ & 1.000 \\
\hline \multirow{2}{*}{14} & Culture & $4.21 \times 10^{7}$ & $<10^{2}$ & 0.008 \\
& qPCR & $9.94 \times 10^{5}$ & $2.58 \times 10^{5}$ & 0.056 \\
\hline \multirow{2}{*}{21} & Culture & $5.40 \times 10^{7}$ & $3.84 \times 10^{7}$ & 0.381 \\
& qPCR & $9.95 \times 10^{5}$ & $9.96 \times 10^{4}$ & 0.032 \\
\hline \multirow{2}{*}{28} & Culture & $9.78 \times 10^{5}$ & $2.93 \times 10^{5}$ & 1.000 \\
& qPCR & $3.53 \times 10^{5}$ & $3.86 \times 10^{5}$ & 1.000 \\
\hline \multirow{2}{*}{35} & Culture & $5.23 \times 10^{5}$ & $2.19 \times 10^{5}$ & 1.000 \\
& qPCR & $2.25 \times 10^{3}$ & $6.95 \times 10^{3}$ & 0.222 \\
\hline \multirow{2}{*}{42} & Culture & $<10^{2}$ & $<10^{2}$ & 1.000 \\
& qPCR & $3.14 \times 10^{4}$ & $*$ & 0.444
\end{tabular}

\footnotetext{
${ }^{1}$ The Mann-Whitney test was used to assess significant differences between controls and animals given supplements $(p<0.05) .{ }^{*}$ Value under the detection limit (one Salmonella spp. genome equivalent/reaction) [20].
}

In 21-day-old challenged broilers, all cecal content challenged was negative for Salmonella spp. either by culture or qPCR. At 7 dpi ( 28 days old), the Salmonella spp. load in the cecum was significantly reduced in the treated group by qPCR $(p=0.016)$, and by culture, which was not significant $(p=0.075)$. At 14 dpi (35 days old), there were no significant differences in the cecal Salmonella spp. load among groups by culture $(p>0.05)$, or by qPCR $(p>0.05)$. Finally, at $21 \mathrm{dpi}$ ( 42 days old) there was a significant reduction in treated broilers by qPCR $(p=0.016)$, not by culture $(p=0.076)$ (Figures 1 and 2, Table 3$)$. 
Table 3. Results of culture (CFU/g) and qPCR (genome equivalent/g) in 21-day-old challenged chickens.

\begin{tabular}{ccccc}
\hline Days-Old & & Control & Treated & $p$-Value ${ }^{\mathbf{1}}$ \\
\hline 7 & Culture & $<10^{2}$ & $<10^{2}$ & 1.000 \\
& qPCR & $*$ & $*$ & 1.000 \\
\hline \multirow{2}{*}{14} & Culture & $<10^{2}$ & $<10^{2}$ & 1.000 \\
& qPCR & $*$ & $*$ & 1.000 \\
\hline \multirow{2}{*}{21} & Culture & $<10^{2}$ & $<10^{2}$ & 1.000 \\
& qPCR & $*$ & $*$ & 1.000 \\
\hline \multirow{2}{*}{28} & Culture & $7.77 \times 10^{7}$ & $2.51 \times 10^{7}$ & 0.075 \\
& qPCR & $2.37 \times 10^{6}$ & $4.68 \times 10^{5}$ & 0.016 \\
\hline \multirow{2}{*}{35} & Culture & $1.49 \times 10^{7}$ & $1.02 \times 10^{5}$ & 0.602 \\
& qPCR & $8.04 \times 10^{5}$ & $4.95 \times 10^{5}$ & 0.310 \\
\hline \multirow{2}{*}{42} & Culture & $7.37 \times 10^{6}$ & $1.35 \times 10^{6}$ & 0.076 \\
& qPCR & $5.18 \times 10^{5}$ & $1.31 \times 10^{5}$ & 0.016
\end{tabular}

${ }^{1}$ The Mann-Whitney test was used to assess significant differences between controls and animals given supplements $(p<0.05) .{ }^{*}$ Value under the detection limit (one Salmonella spp. genome equivalent/reaction) [20].

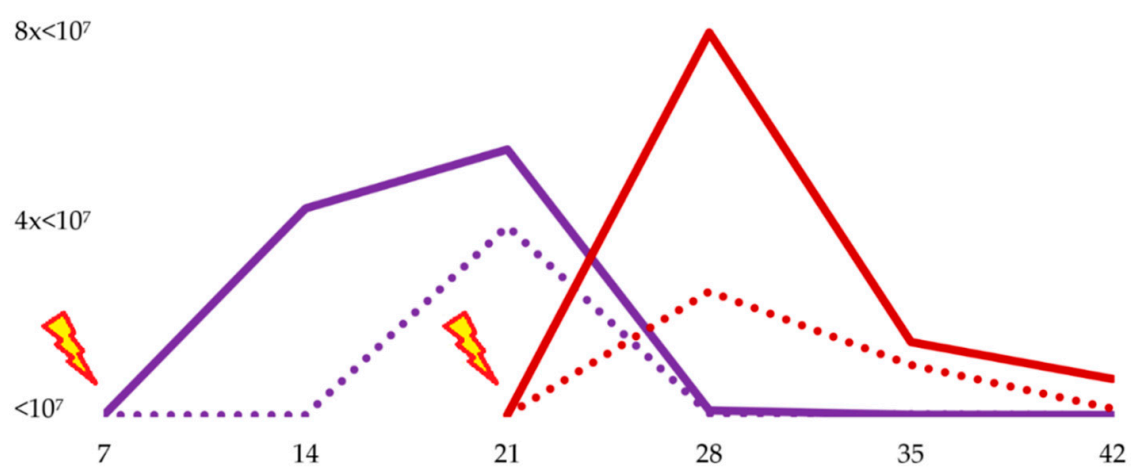

Figure 1. Results of the Salmonella spp. count in selective agar in broilers challenged with Salmonella Typhimurium at day 7 (purple lines) and day 21 (red lines) of life. Continuous lines represent control groups, whereas discontinuous lines represent treated groups, fed with fermented defatted 'alperujo'. The Salmonella spp. count by culture (CFU/g) is shown on the vertical axis, and samplings $(7,14,21,35$, or 42) on the horizontal axis.

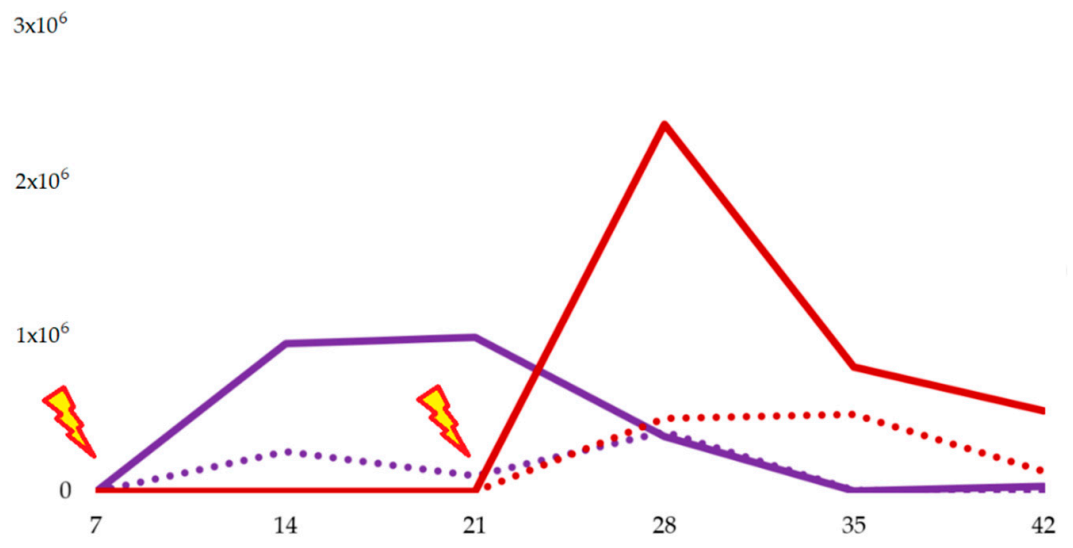

Figure 2. Results of the qPCR for Salmonella spp. in broilers challenged with Salmonella Typhimurium at day 7 (purple lines) and day 21 (red lines) of life. Continuous lines represent control groups, whereas discontinuous lines represent treated groups, fed with fermented defatted 'alperujo'. The genome equivalent/g is shown on the vertical axis, and samplings $(7,14,21,35$, or 42$)$ on the horizontal axis. 
The number of positive chickens by culture and qPCR at both challenge times is detailed in Table 4 .

Table 4. Number of animals testing positive for Salmonella spp. by culture and qPCR.

\begin{tabular}{cccccccc}
\hline & & \multicolumn{2}{c}{ 7-Day-Old Challenged } & \multicolumn{3}{c}{ 21-Day-Old Challenged } \\
\hline \multirow{2}{*}{ Days-Old } & & Control & Treated & $p$-Value & Control & Treated & $p_{\text {-Value }}$ 1 $^{\mathbf{1}}$ \\
\hline \multirow{2}{*}{7} & Culture & $0 / 5$ & $0 / 5$ & 2 & $0 / 5$ & $0 / 5$ & 2 \\
& qPCR & $0 / 5$ & $0 / 5$ & 2 & $0 / 5$ & $0 / 5$ & 2 \\
\hline \multirow{2}{*}{14} & Culture & $5 / 5$ & $0 / 5$ & $>0.05$ & $0 / 5$ & $0 / 5$ & 2 \\
& qPCR & $5 / 5$ & $4 / 5$ & $>0.05$ & $0 / 5$ & $0 / 5$ & 2 \\
\hline \multirow{2}{*}{21} & Culture & $5 / 5$ & $3 / 5$ & $>0.05$ & $0 / 5$ & $0 / 5$ & 2 \\
& qPCR & $5 / 5$ & $4 / 5$ & $>0.05$ & $0 / 5$ & $0 / 5$ & 2 \\
\hline \multirow{2}{*}{28} & Culture & $3 / 5$ & $3 / 5$ & $>0.05$ & $5 / 5$ & $3 / 5$ & $>0.05$ \\
& qPCR & $5 / 5$ & $5 / 5$ & 2 & $5 / 5$ & $5 / 5$ & 2 \\
\hline \multirow{2}{*}{35} & Culture & $2 / 5$ & $2 / 5$ & $>0.05$ & $5 / 5$ & $5 / 5$ & 2 \\
& qPCR & $5 / 5$ & $5 / 5$ & $>0.05$ & $5 / 5$ & $5 / 5$ & 2 \\
\hline \multirow{2}{*}{42} & Culture & $0 / 5$ & $0 / 5$ & 2 & $5 / 5$ & $5 / 5$ & 2 \\
\hline
\end{tabular}

${ }^{1}$ The Fisher's exact test was used to assess significant differences between control and treated animals. ${ }^{2}$ No statistics are computed because the value obtained is a constant.

\subsection{Intestinal Histopathology}

In both control groups, in 7- or 21-day-old challenge, the duodenum at 7 dpi (14 or 28 days of life) displayed a moderate atrophy and stunting of the villi with mild epithelial desquamation and a slight increase in intraepithelial lymphocytes. The lamina propria was moderately to severely expanded by an inflammatory infiltrate composed of lymphocytes, heterophils, and macrophages that partially distorted the crypt structure (Figure 3a). At 14 dpi (21 or 35 days of life), in the control group the lesions were similar, with additional mild crypt distortion and mild gut-associated lymphoid tissue (GALT) hyperplasia. In the treated group, there was a reduction in the severity of villous stunting and lymphocytic infiltrate (Figure 3b).

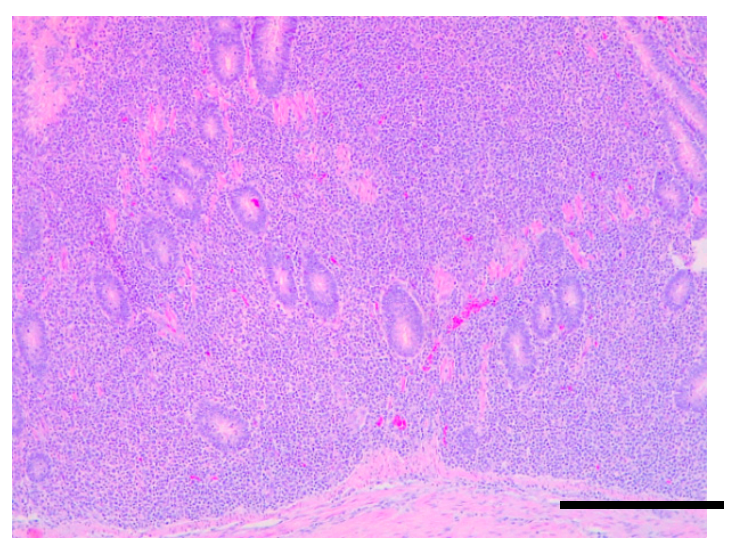

(a)

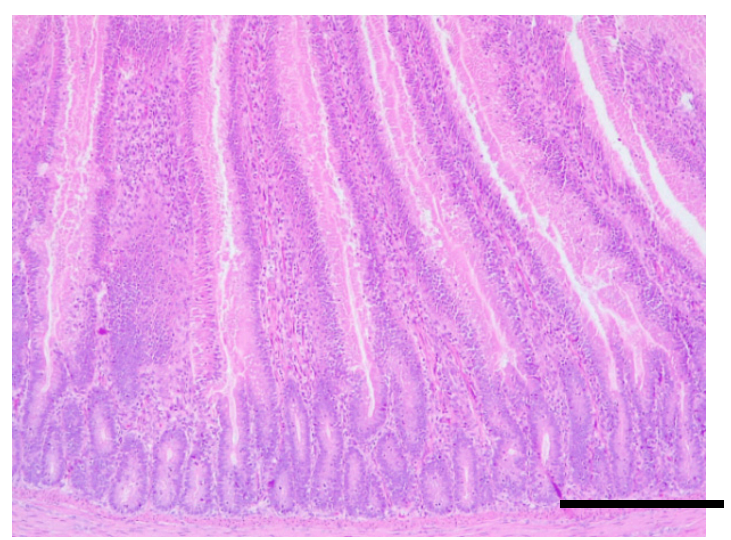

(b)

Figure 3. Histopathological study of the duodenum of 14-day-old broilers, 7 days post-infection with Salmonella Typhimurium. (a) Control group. There is a severe lymphocytic inflammatory infiltrate that completely distorted the crypt structure. There are scattered haemorrhagic foci. (b) Treated group. The lamina propria is mildly expanded by a lymphocytic inflammatory infiltrate with a few heterophils. 20x, scale bar: $250 \mu \mathrm{m}$. 
In the cecum, at $7 \mathrm{dpi}$ (14 or 28 days of life), control groups presented a mild epithelial desquamation and the lamina propria was slightly expanded by an infiltrate composed of lymphocytes and plasma cells that moderately to severely distorted the crypt structure. GALT hyperplasia was moderate to severe (Figure 4a). By 14 dpi (21 or 35 days of life), all those changes were maintained in both control groups with an additional increase in intraepithelial lymphocytes. In the treated group, there was a reduction in the intensity of lamina propria lymphocytic infiltration and GALT hyperplasia compared to the control group (Figure $4 \mathrm{~b}$ ).

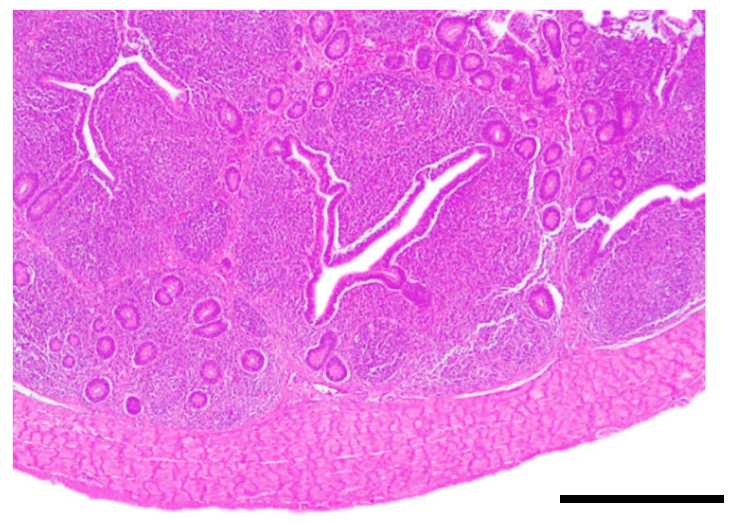

(a)

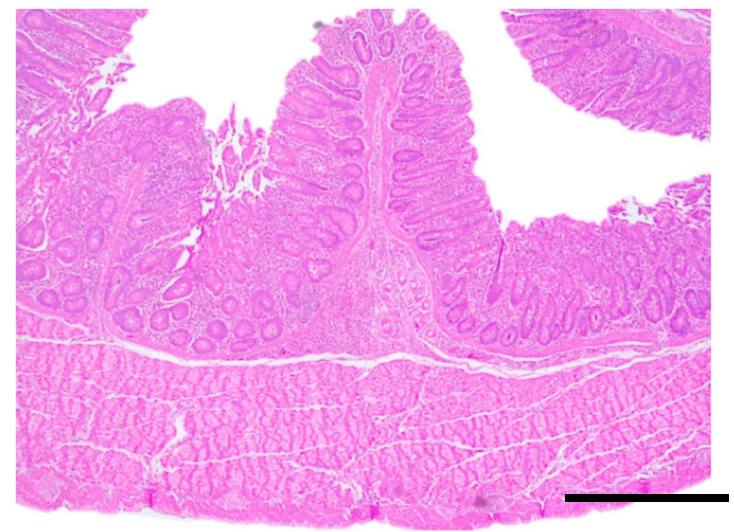

(b)

Figure 4. Histopathological study of the cecum of 14-day-old broilers, 7 days post-infection with Salmonella Typhimurium. (a) Control group. There is a severe lymphocytic inflammatory infiltrate that completely distorted the crypt structure. GALT hyperplasia is evident. (b) Treated group. The lamina propria is mildly expanded by a lymphocytic inflammatory infiltrate. $20 \times$, scale bar: $250 \mu \mathrm{m}$.

\subsection{Intestinal Morphology}

In 7-day-old challenged chickens, duodenum villi height was significantly improved in treated chickens on days $7,14,28,35$, and $42(p<0.05)$. Similarly, the crypts in the duodenum were deeper in in all treated samplings $(p<0.05)$. Regarding ceca morphology, crypts were seen to be deeper in 7-, 21and 42-day-old treated chickens $(p<0.05)$. At 28 days of life, controls displayed a higher value for crypt depth $(p<0.05)$ (Table 5).

Broilers in the treated group challenged at 21 days of age showed a significant improvement in the duodenum villi height at 28,35 , and 42 days of life $(p<0.05)$. The depth of the crypts in the duodenum was significantly improved by the treatment on days 28 and $42(p<0.05)$. The cecum crypt was deeper in treated chickens on days 35 and 42 of life $(p<0.05)$ (Table 5).

\subsection{Cecal Microbiota}

In chickens challenged at 7 days of age, there were no statistically-significant differences among the groups established. The most abundant bacterial family at days 7, 14, and 21 of life was Enterobacteriaceae in both groups. At day 28, Enterobacteriaceae drastically decreased and was replaced by Lachnospiraceae and Ruminococcaceae in similar abundance, and these were also the most prevalent families at 35 days of life, with a higher abundance of Lachnospiraceae. Finally, at day 42 of life, Bacteroidaceae, Ruminococcaceae, and Lachnospiraceae were the most prevalent families in both groups (Figure 5).

In chickens challenged at 21 days of age, there were no statistically-significant differences among the groups established. The most abundant bacterial family at day 7 was Enterobacteriaceae in the control group and Lactobacillaceae in the treated group. At day 14 of life, Enterobacteriaceae was still prevalent in the control group, whereas in the treated group Enterobacteriaceae and Ruminococcaceae were more abundant, being substituted by Lachnospiraceae as the second-most-abundant family in both groups at 21 days of life. In 28-day-old broilers, Lactobacillaceae and Ruminococcaceae were the most 
abundant families in the control group, whereas Lachnospiraceae and Ruminococcaceae were predominant in treated chickens. On days 35 and 42 of life, Ruminococcaceae and Lachnospiraceae were the most abundant families, with slightly higher values of Ruminococcaceae over Lachnospiraceae in the control groups (Figure 6).

Table 5. Histomorphometric results: duodenum villi height, duodenum crypt depth and ceca crypt depth. Number of samples (N), mean values (mean), standard deviation (SD), and $p$-value are detailed per day of life and challenge group.

\begin{tabular}{|c|c|c|c|c|c|c|c|}
\hline & \multicolumn{3}{|c|}{ Control } & \multicolumn{3}{|c|}{ Supplemented } & \multirow{2}{*}{$p$-Value } \\
\hline & $\mathbf{N}$ & Mean & SD & $\mathbf{N}$ & Mean & SD & \\
\hline \multicolumn{8}{|c|}{ 7-Day-Old Challenge } \\
\hline \multicolumn{8}{|c|}{ Duodenum Villus Heights } \\
\hline 7 days & 100 & 539.81 & 192.04 & 100 & 853.62 & 217.81 & $<0.001$ \\
\hline 14 days & 100 & 896.95 & 193.71 & 100 & 1028.75 & 398.60 & 0.006 \\
\hline 21 days & 100 & 1208.45 & 281.81 & 100 & 1188.42 & 202.38 & 0.767 \\
\hline 28 days & 100 & 1118.62 & 225.74 & 100 & 1200.56 & 246.92 & 0.011 \\
\hline 35 days & 100 & 893.23 & 203.78 & 100 & 1258.92 & 269.51 & $<0.001$ \\
\hline 42 days & 100 & 923.01 & 225.90 & 100 & 1310.98 & 241.50 & $<0.001$ \\
\hline \multicolumn{8}{|c|}{ Duodenum Crypt Depths } \\
\hline 7 days & 100 & 78.14 & 16.49 & 100 & 89.16 & 26.49 & 0.001 \\
\hline 14 days & 100 & 93.91 & 29.84 & 100 & 131.74 & 134.48 & $<0.001$ \\
\hline 21 days & 100 & 128.09 & 49.01 & 100 & 141.61 & 44.84 & 0.005 \\
\hline 28 days & 100 & 137.74 & 39.52 & 100 & 153.28 & 35.11 & 0.001 \\
\hline 35 days & 100 & 124.56 & 37.50 & 100 & 151.71 & 38.22 & $<0.001$ \\
\hline 42 days & 100 & 107.82 & 37.22 & 100 & 122.56 & 39.46 & 0.012 \\
\hline \multicolumn{8}{|c|}{ Cecum Crypt Depths } \\
\hline 7 days & 100 & 181.72 & 61.43 & 100 & 227.93 & 105.39 & 0.002 \\
\hline 14 days & 100 & 266.79 & 76.54 & 100 & 288.37 & 90.69 & 0.189 \\
\hline 21 days & 100 & 204.25 & 71.28 & 100 & 235.64 & 91.31 & 0.028 \\
\hline 28 days & 100 & 276.78 & 69.49 & 100 & 266.57 & 109.68 & 0.006 \\
\hline 35 days & 100 & 234.47 & 65.44 & 100 & 249.76 & 78.30 & 0.208 \\
\hline 42 days & 100 & 233.19 & 67.96 & 100 & 353.70 & 165.79 & $<0.001$ \\
\hline \multicolumn{8}{|c|}{ 21-Day-Old Challenge } \\
\hline \multicolumn{8}{|c|}{ Duodenum Villus Heights } \\
\hline 28 days & 100 & 1052.40 & 323.90 & 100 & 1353.79 & 220.63 & $<0.001$ \\
\hline 35 days & 100 & 1039.11 & 233.53 & 100 & 1193.80 & 195.27 & $<0.001$ \\
\hline 42 days & 100 & 888.01 & 200.58 & 100 & 1210.86 & 233.54 & $<0.001$ \\
\hline \multicolumn{8}{|c|}{ Duodenum Crypt Depths } \\
\hline 28 days & 100 & 145.07 & 45.43 & 100 & 158.67 & 47.70 & 0.033 \\
\hline 35 days & 100 & 125.94 & 35.36 & 100 & 133.36 & 33.81 & 0.179 \\
\hline 42 days & 100 & 120.93 & 32.62 & 100 & 154.44 & 36.30 & $<0.001$ \\
\hline \multicolumn{8}{|c|}{ Cecum Crypt Depths } \\
\hline 28 days & 100 & 298.80 & 144.55 & 100 & 272.91 & 116.46 & 0.354 \\
\hline 35 days & 100 & 230.23 & 128.98 & 100 & 325.47 & 236.05 & $<0.001$ \\
\hline 42 days & 100 & 238.19 & 98.04 & 100 & 409.40 & 246.84 & $<0.001$ \\
\hline
\end{tabular}

${ }^{1}$ The Mann-Whitney test was used to assess significant differences between controls and animals given supplements $(p<0.05)$. 


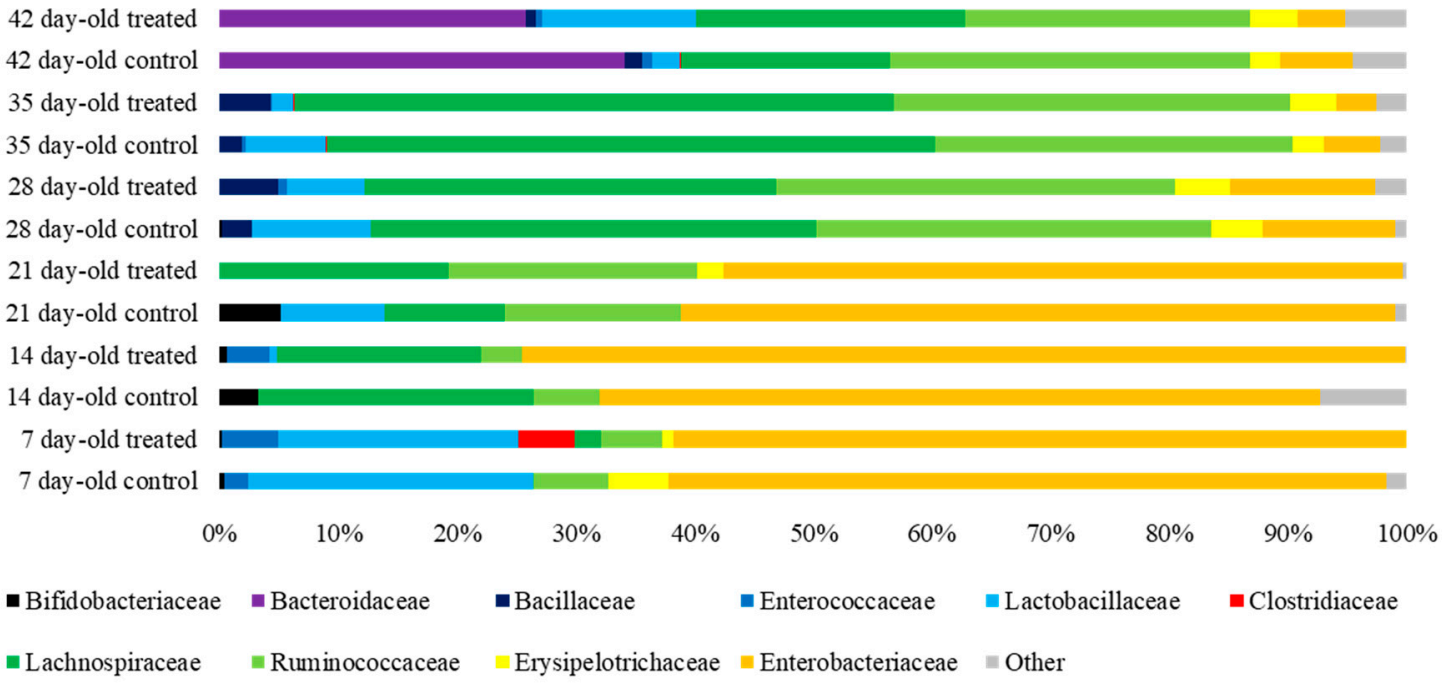

Figure 5. Bar chart showing the 10 most abundant bacterial families found in the cecal content of control and treated broiler chickens challenged with Salmonella Typhimurium at 7 days of life. Each bar represents the relative abundance (horizontal axis) of bacterial families by group of animals, diet treatment, and age.

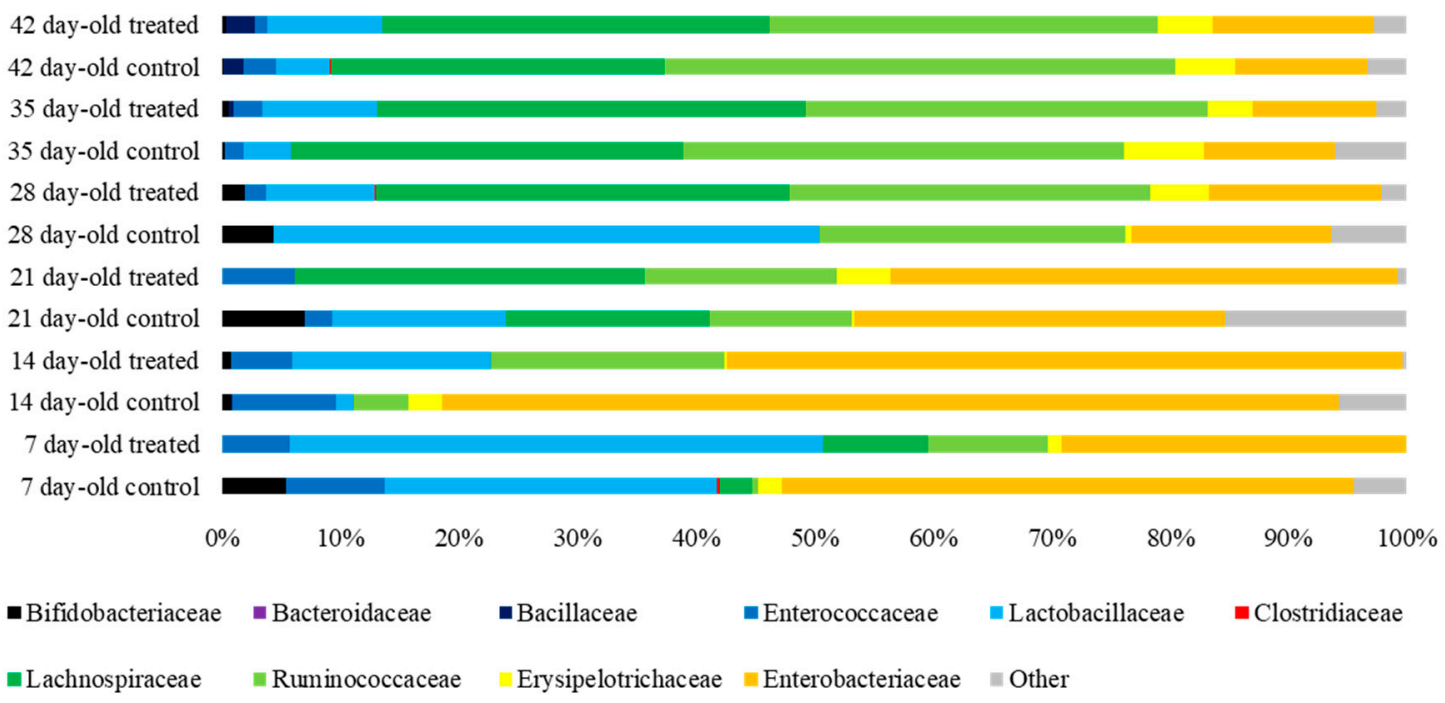

Figure 6. Bar chart showing the 10 most abundant bacterial families found in the cecal content of control and treated broiler chickens challenged with Salmonella Typhimurium at 21 days of life. Each bar represents the relative abundance (horizontal axis) of bacterial families by group of animals, diet treatment, and age.

\section{Discussion}

A reduction of the intestinal colonisation of Salmonella spp. in broilers may contribute to reducing bacterial shedding in the environment, thus avoiding transmission in the farm and contamination of poultry products through the food chain [26,27]. Salmonella spp. infection in chickens usually occurs in the early stages due to their impaired immunity $[7,28,29]$. We have proposed an infection in 7 or 21-day-old chickens with weekly samplings to evaluate the dynamics of infection in control and treated animals. The cecum is known to be the main site of colonisation for Salmonella spp. [27,28], and therefore fresh feces were collected from this segment for analysis. Culture- and qPCR-positive results confirmed that a Salmonella Typhimurium infection was established. The control group showed an 
initial increase in the Salmonella spp. count in the cecum until 14 dpi, followed by a rapid decrease, as expected according to previous studies [7,27-29]. Other authors observed a high rate of persistent infection in older chickens infected in the first week of life [30], although this could be explained by the high inoculation dose [28]. In 7-day-old challenged chickens, we observed a significant reduction of Salmonella spp. in the treated group at $7 \mathrm{dpi}$ by culture and $14 \mathrm{dpi}$ by qPCR analysis. Additionally, the number of positive chickens was significantly lower in the treated group at $7 \mathrm{dpi}$. These results may indicate that supplementation of poultry diet with FDA may delay and reduce Salmonella Typhimurium colonisation in the cecum of young broilers. A similar reduction of $S$. Typhimurium in the cecum of challenged broilers given supplements of fermented soybean has been reported [2].

The age of infection is known to influence pathogenesis in avian salmonellosis [31]. It is believed that a high dose is necessary to infect older chickens [28], although we maintained the same dose to allow comparison between the two challenge periods. Interestingly, we obtained higher values based on culture data for controls challenged at 21 days of life compared to 7-day-old controls, even if it is known that chickens over 3 weeks old are less susceptible to Salmonella spp. [31]. Moreover, we have observed a shortening time in the reduction of Salmonella spp. to low rates in 21-day-old challenged chicks compared to 7-day-old ones. In fact, it has been reported that 3-week-old broiler infection, as performed here, resulted in animals being infected for two weeks as the resolution of the Salmonella spp. infection occurred faster in old challenged chickens [26]. This could be partially explained by the increased immunity in older broilers as the age of infection seemed to influence Salmonella spp. persistence in the ceca [30]. Older animals have a more mature microbiota that, in our case, could provide colonization resistance and cause less colonization of pathogens, like Salmonella. In the treated group challenged at 21 days of life, we observed a reduction in the Salmonella spp. count by qPCR at $7 \mathrm{dpi}$, which is similar to what we observed in 7-day-old challenged chicks. This reduction was also confirmed by traditional culture, which reveals that FDA may also reduce Salmonella spp. colonisation in older newly-infected broilers. We agree with previous studies that infection, regardless of chicken age, resulted in the presence of Salmonella spp. in the intestine at slaughter age (42 days-old) [30]. Nevertheless, we observed a significant reduction of the Salmonella spp. carriage in the cecum of 21-day-old challenged animals of the treated group by qPCR at 42 days of life. Thus, FDA may help to reduce Salmonella spp. in the cecum of broilers and therefore contribute to diminishing carcass contamination at slaughter.

The effect of natural phenolic compounds has not been previously tested in an avian challenge model of Salmonella Typhimurium. The bioactive molecules like polyphenols contained in olive oil extracts are known to show marked antimicrobial activity against Salmonella spp. in concentrations as high as $5 \times 10^{5} \mathrm{CFU} / \mathrm{mL}$ [12]. Even if their concentration in extracts is notably superior to those found in olive oil and their by-products [12], the synergistic action of the dialdehyde form of decarboxymethyl ligstroside, oleuropein aglycons, hydroxytyrosol, and tyroxol partially compensates this matter and retains antimicrobial proprieties [10]. The delaying and reduction of Salmonella Typhimurium colonisation in the cecum in the present study could be explained by the antimicrobial effects of phenols and polyphenols present in the compound tested $[8,14]$. Specifically, hydroxytyrosol has been reported to be more active against Salmonella spp. compared to oleuropein [13]. These effects on Salmonella spp. in vitro are bacteriostatic, inhibiting the division by reducing intracellular concentration of ATP and depolarising the cell membrane leading to bacterial death [8]. In addition, FDA also contains a significant proportion of fibre [14,15], which has been suggested to prevent pathogen adhesion to the intestinal surface [26].

Intestinal mucosa evaluation can provide information on health status in poultry as stressors such as enteric infections may lead to structural modifications $[6,32,33]$. We found similar histopathological features regardless of the age of infection in both the duodenum and cecum of broilers. These findings are in line with studies assessing $S$. Typhimurium-induced histopathology in chickens $[29,34,35]$. The inflammatory infiltrate observed was predominantly composed of lymphocytes and severely affected the duodenum. In fact, the duodenum has been reported to be intensively affected after 
a Salmonella spp. challenge, eliciting an immune response composed mainly of mononuclear cells (T-lymphocytes) and heterophils [36-38]. Furthermore, we found that supplementation with FDA slightly reduced the severity of the lesions in the treated group, which paralleled what has been reported after supplementation with arginine in the jejunum of broilers [35].

Many authors have reported shortening of villi after a Salmonella spp. challenge [2,6,7,29,35,39-43], which implies difficulty in absorption capacity and a reduction in body weight gain $[29,40,41,44]$. In our study, we were able to observe a significant increase in the duodenum villi height of the treated group in most samplings, as previously described in healthy broilers [15]. A similar increase in villus height in the jejunum of broilers given supplements of probiotics or prebiotics and challenged with S. Typhimurium has been reported $[2,6,32,40,42,44,45]$. Fermented soybean, butyric acid, sodium butyrate, oligosaccharides, zinc, and dietary clay supplementation in $S$. Typhimurium-challenged broilers resulted in an increase in villi height in the small intestine, mitigating Salmonella spp. colonisation effects $[2,6,32,40,42,44]$. Similarly, carvacrol essential oil has showed a protective effect in the villus structure against a Campylobacter spp. infection in broilers [45]. The increase in the height of the villi observed here contributes to enhancing the absorption capacity of the intestine in animals infected with Salmonella spp. [7,32], helping to palliate the functional compromise in digestion, transport, and absorption in the alimentary tract [42].

The immune system of young chicks is not fully competent until weeks after hatching [30], which is why they are more prone to developing systemic infections. Thus, unspecific immune response is important in pathogen clearance and response to damage in the intestine. In our study, duodenal and cecal crypts were deeper in the treated group in almost all samplings. It has been previously reported that FDA stimulates crypt growth in healthy broilers [15], thus safeguarding epithelial renewal. Epithelial turnover has shown to be fundamental to the response to insults to the superficial mucosa [14], as a rapid epithelial replication promotes a quick healing of superficial lesions. After Salmonella spp. infection, an increase in the depth of the crypts is expected to compensate superficial mucosal damage [6,29,41]. Intestinal microbiota contributes to host susceptibility to infections [43]. Sequencing data of the $16 \mathrm{~S}$ rDNA showed that the population up to 21 days of life was composed mainly by Enterobacteriaceae, which drastically decreased thereafter [46,47]. Enterobacteriaceae has been reported as the most abundant bacterial family in S. Typhimurium-challenged broilers [35]. We observed a reduction in Enterobacteriaceae in treated groups in several samplings. Such reduction of Enterobacteriaceae in the chicken gut has been associated with an increase in small chain fatty acid production [47], so the reduction of Enterobacteriaceae after FDA consumption may, to some extent, stimulate small chain fatty acid production. It has been described that increased production of small chain fatty acid in the avian intestine may lead to an increase in epithelial renewal as well as in villi height [2]. Moreover, the small chain fatty acid butyrate has been reported to reduce Salmonella spp. colonisation in the cecum [44].

In 7-day-old challenged chickens, Enterobacteriaceae was replaced by Lachnospiraceae and Ruminococcaceae from 28 to 35 days of life as previously reported $[47,48]$. The functions of Lachnospiraceae and Ruminococcaceae involve the production of short chain fatty acids through the fermentation of indigestible polysaccharides $[47,49]$. In addition, Lachnospiraceae possess hydrolases that allow the digestion of starch and glycogen by means of the rupture of $\alpha$-amylase bonds [47]. Lachnospiraceae and Ruminococcaceae have been reported to diminish their abundance in inflammatory processes due to reactive oxygen species production by inflammatory cells [49]. We observed a reduction in both families a week after infection in 7-day-old challenged chicks. However, in the 21-day-old challenge, we found an increase in both Lachnospiraceae and Ruminococcaceae in the treated group during the two weeks following the challenge. In 21-day-old challenged broilers, one week post-infection, Lactobacillaceae and Ruminococcaceae were the most abundant families in the treated group. Lactobacillaceae are carbohydrate fermenters, contributing to the production of short chain fatty acids in the poultry intestine [49], which suggests that there may be beneficial microbiota variations in the treated group. Moreover, 
S. Typhimurium infection is known to reduce Lactobacillaceae in the chicken gut [40], so fermented defatted 'alperujo' may also contribute to mitigating disbiosis in infected chickens.

\section{Conclusions}

Dietary supplementation with fermented defatted 'alperujo' (FDA) was, to some extent, effective in delaying and reducing Salmonella Typhimurium colonisation in the cecum after a 7- or 21-day-old challenge. The significant reduction observed in the cecum in 42-day-old broilers may suggest that FDA stimulates Salmonella Typhimurium clearance in the cecum and may contribute to diminishing the risk of carcass contamination at slaughter. Additionally, the improvement in mucosal integrity suggests that enhancing intestinal health helps to mitigate Salmonella spp. infection in the host, and production losses. Microbiota composition variations after supplementation may be beneficial and may help to prevent dysbiosis. These results could provide evidence that this olive oil by-product would contribute to prophylactic and therapeutic measures to reduce salmonellosis prevalence in poultry farms.

Author Contributions: Conceptualisation, L.D. and A.R.-B.; methodology, A.R.-M., M.U.-R., M.H., P.M.-V., D.A. and D.R.-L.; software, A.R.-M., M.U.-R. and M.H.; validation, M.U.-R., M.H., D.R.-L., L.d.J., L.D. and A.R.-B.; formal analysis, A.R.-M., M.U.-R. and M.H.; investigation, A.R.-M. and M.U.-R.; resources, D.R.-L., L.d.J., L.D. and A.R.-B.; data curation, A.R.-M., M.U.-R. and M.H.; writing-original draft preparation, A.R.-M.; writing-review and editing, M.U.-R. and A.R.-B.; visualisation, L.D. and A.R.-B.; supervision, M.U.-R. and A.R.-B.; project administration, M.U.-R., L.d.J. and A.R.-B.; funding acquisition, L.d.J. and L.D. All authors have read and agreed to the published version of the manuscript.

Funding: This research was funded by the European Union's Horizon 2020 research and innovation programme, grant number 773830 .

Acknowledgments: This study was conducted as part of the project "Promoting One Health in Europe through Joint Actions on Foodborne Zoonoses, Antimicrobial Resistance, and Emerging Microbiological Hazards (ONE HEALTH EJP)". We would like to thank Luis Arrabal (Porres y Barios, S.A., Córdoba, Spain) for providing the fermented defatted 'alperujo', VISAVET Health Surveillance Centre personnel involved in the project (M. C. Jiménez, S. Cruz, G. Torre, F. Mayoral, N. Porras, L. Barreno, M. García, E. Rivero, N. Maasoumi, M. Mazariegos, L. Delgado. D. Duque, and P. Alcubilla), P. Cuesta-Álvaro (Complutense University of Madrid) for statistical analysis, and English language services provided by Bill Newton (Complutense University of Madrid).

Conflicts of Interest: The authors declare no conflict of interest.

\section{References}

1. EFSA. The European Union summary reports on trends and sources of zoonoses, zoonotic agents and food-borne outbreaks in 2016. EFSA J. 2017, 15, 5077. [CrossRef]

2. Jazi, V.; Mohebodini, H.; Ashayerizadeh, A.; Shabani, A.; Barekatain, R. Fermented soybean meal ameliorates Salmonella Typhimurium infection in young broiler chickens. Poult. Sci. 2019, 98, 5648-5660. [CrossRef] [PubMed]

3. Fasina, Y.O.; Bowers, J.B.; Hess, J.B.; McKee, S.R. Effect of dietary glutamine supplementation on Salmonella colonization in the ceca of young broiler chicks. Poult. Sci. 2010, 89, 1042-1048. [CrossRef]

4. Awad, W.A.; Aschenbach, J.R.; Khayal, B.; Hess, C.; Hess, M. Intestinal epithelial responses to Salmonella enterica serovar Enteritidis: Effects on intestinal permeability and ion transport. Poult. Sci. 2012, 91, 2949-2957. [CrossRef] [PubMed]

5. Sibanda, N.; McKenna, A.; Richmond, A.; Ricke, S.C.; Callaway, T.; Stratakos, A.C.; Gundogdu, O.; Corcionivoschi, N. A review of the effect of management practices on campylobacter prevalence in poultry farms. Front. Microbiol. 2018, 9, 2002. [CrossRef] [PubMed]

6. Jazi, V.; Foroozandeh, A.D.; Toghyani, M.; Dastar, B.; Rezaie-Koochaksaraie, R.; Toghyani, M. Effects of Pediococcus acidilactici, mannan-oligosaccharide, butyric acid and their combination on growth performance and intestinal health in young broiler chickens challenged with Salmonella Typhimurium. Poult. Sci. 2018, 97, 2034-2043. [CrossRef] [PubMed] 
7. Abudabos, A.M.; Hussein, E.O.S.; Ali, M.H.; Al-Ghadi, M.Q. The effect of some natural alternative to antibiotics on growth and changes in intestinal histology in broiler exposed to Salmonella challenge. Poult. Sci. 2018, 98, 1441-1446. [CrossRef]

8. Guo, L.; Gong, S.; Wang, Y.; Sun, Q.; Duo, K.; Fei, P. Antibacterial activity of olive oil polyphenol extract against Salmonella Typhimurium and Staphylococcus aureus: Possible Mechanisms. Foodborne Pathog. Dis. 2019, 17, 396-403. [CrossRef]

9. Alburquerque, J.A.; Gonzálvez, J.; García, D.; Cegarra, J. Agrochemical characterisation of "alperujo”, a solid by-product of the two-phase centrifugation method for olive oil extraction. Bioresour. Technol. 2004, 91, 195-200. [CrossRef]

10. Medina, E.; Romero, C.; Brenes, M.; de Castro, A. Antimicrobial activity of olive oil, vinegar, and various beverages against foodborne pathogens. J. Food Prot. 2007, 70, 1194-1199. [CrossRef]

11. Herrero-Encinas, J.; Blanch, M.; Pastor, J.J.; Mereu, A.; Ipharraguerre, I.R.; Menoyo, D. Effects of a bioactive olive pomace extract from Olea europaea on growth performance, gut function, and intestinal microbiota in broiler chickens. Poult. Sci. 2020, 99, 2-10. [CrossRef] [PubMed]

12. Karaosmanoglu, H.; Soyer, F.; Ozen, B.; Tokatli, F. Antimicrobial and antioxidant activities of Turkish extra virgin olive oils. J. Agric. Food Chem. 2010, 58, 8238-8245. [CrossRef] [PubMed]

13. Bisignano, G.; Tomaino, A.; Cascio, R.L.; Crisafi, G.; Uccella, N.; Saija, A. On the In-vitro antimicrobial activity of oleuropein and hydroxytyrosol. J. Pharm. Pharmacol. 1999, 51, 971-974. [CrossRef] [PubMed]

14. Rebollada-Merino, A.; Bárcena, C.; Ugarte-Ruiz, M.; Porras, N.; Mayoral-Alegre, F.J.; Tomé-Sánchez, I.; Domínguez, L.; Rodríguez-Bertos, A. Effects on intestinal mucosal morphology, productive parameters and microbiota composition after supplementation with fermented defatted alperujo (FDA) in laying hens. Antibiotics 2019, 8, 215. [CrossRef]

15. Rebollada-Merino, A.; Ugarte-Ruiz, M.; Hernández, M.; Miguela-Villoldo, P.; Abad, D.; Cuesta-Álvaro, P.; Rodríguez-Lázaro, D.; de Juan, L.; Domínguez, L.; Rodríguez-Bertos, A. Dietary supplementation with fermented defatted "alperujo" induces modifications of the intestinal mucosa and cecal microbiota of broiler chickens. Poult. Sci. 2020. online ahead of print. [CrossRef]

16. Azcarate-Peril, M.A.; Butz, N.; Cadenas, M.B.; Koci, M.; Ballou, A.; Mendoza, M.; Ali, R.; Hassan, H. An attenuated Salmonella enterica serovar Typhimurium strain and galacto-oligosaccharides accelerate clearance of Salmonella infections in poultry through modifications to the gut microbiome. Appl. Environ. Microbiol. 2017, 84, e02526-17. [CrossRef] [PubMed]

17. Asociación Española de Normalización (UNE). Available online: https://www.une.org/encuentra-tu-norma/ busca-tu-norma/norma?c=N0058760 (accessed on 15 September 2020).

18. Miguela-Villoldo, P.; Hernández, M.; Moreno, M.A.; Rodríguez-Lázaro, D.; Quesada, A.; Domínguez, L.; Ugarte-Ruiz, M. National colistin sales versus colistin resistance in Spanish pig production. Res. Vet. Sci. 2019, 123, 141-143. [CrossRef]

19. Rodríguez-Lázaro, D.; Gonzalez-García, P.; Delibato, E.; De Medici, D.; García-Gimeno, R.M.; Valero, A.; Hernandez, M. Next day Salmonella spp. detection method based on real-time PCR for meat, dairy and vegetable food products. Int. J. Food Microbiol. 2014, 184, 113-120. [CrossRef]

20. Rodríguez-Lázaro, D.; Hernández, M.; Esteve, T.; Hoorfar, J.; Pla, M. A rapid and direct real time PCR-based method for identification of Salmonella spp. J. Microbiol. Methods 2003, 54, 381-390. [CrossRef]

21. Klindworth, A.; Pruesse, E.; Schweer, T.; Peplies, J.; Quast, C.; Horn, M.; Glöckner, F.O. Evaluation of general 16S ribosomal RNA gene PCR primers for classical and next-generation sequencing-based diversity studies. Nucleic Acids Res. 2013, 41, e1. [CrossRef]

22. Bolyen, E.; Rideout, J.R.; Dillon, M.R.; Bokulich, N.A.; Abnet, C.C.; Al-Ghalith, G.A.; Alexander, H.; Alm, E.J.; Arumugam, M.; Asnicar, F.; et al. Reproducible, interactive, scalable and extensible microbiome data science using QIIME 2. Nat. Biotechnol. 2019, 37, 852-857. [CrossRef] [PubMed]

23. Callahan, B.J.; McMurdie, P.J.; Rosen, M.J.; Han, A.W.; Johnson, A.J.A.; Holmes, S.P. DADA2: High-resolution sample inference from Illumina amplicon data. Nat. Methods 2016, 13, 581-583. [CrossRef] [PubMed]

24. Wang, Q.; Garrity, G.M.; Tiedje, J.M.; Cole, J.R. Naive Bayesian classifier for rapid assignment of rRNA sequences into the new bacterial taxonomy. Appl. Environ. Microbiol. 2007, 73, 5261-5267. [CrossRef]

25. Quast, C.; Pruesse, E.; Yilmaz, P.; Gerken, J.; Schweer, T.; Yarza, P.; Peplies, J.; Glöckner, F.O. The SILVA ribosomal RNA gene database project: Improved data processing and web-based tools. Nucleic Acids Res. 2013, 41, 590-596. [CrossRef] 
26. Faber, T.A.; Dilger, R.N.; Iakiviak, M.; Hopkins, A.C.; Price, N.P.; Fahey, G.C. Ingestion of a novel galactoglucomannan oligosaccharide-arabinoxylan (GGMO-AX) complex affected growth performance and fermentative and immunological characteristics of broiler chicks challenged with Salmonella Typhimurium. Poult. Sci. 2012, 91, 2241-2254. [CrossRef] [PubMed]

27. Menanteau, P.; Kempf, F.; Trotereau, J.; Virlogeux-Payant, I.; Gitton, E.; Dalifard, J.; Gabriel, I.; Rychlik, I.; Velge, P. Role of systemic infection, cross contaminations and super-shedders in Salmonella carrier state in chicken. Environ. Microbiol. 2018, 20, 3246-3260. [CrossRef] [PubMed]

28. Bjerrum, L.; Engberg, R.M.; Pedersen, K. Infection models for Salmonella Typhimurium DT110 in day-old and 14-day-old broiler chickens kept in isolators. Avian Dis. 2003, 47, 1474-1480. [CrossRef]

29. Fasina, Y.O.; Hoerr, F.J.; McKee, S.R.; Conner, D.E. Influence of Salmonella enterica serovar Typhimurium infection on intestinal goblet cells and villous morphology in broiler chicks. Avian Dis. 2010, 54, 841-847. [CrossRef]

30. Beal, R.; Wigley, P.; Powers, C.; Hulme, S.; Barrow, P.; Smith, A. Age at primary infection with Salmonella enterica serovar Typhimurium in the chicken influences persistence of infection and subsequent immunity to re-challenge. Vet. Immunol. Immunopathol. 2004, 100, 151-164. [CrossRef]

31. Marcq, C.; Cox, E.; Szalo, I.M.; Thewis, A.; Beckers, Y. Salmonella Typhimurium oral challenge model in mature broilers: Bacteriological, immunological, and growth performance aspects. Poult. Sci. 2010, 90, 59-67. [CrossRef]

32. Shang, Y.; Regassa, A.; Kim, J.H.; Kim, W.K. The effect of dietary fructooligosaccharide supplementation on growth performance, intestinal morphology, and immune responses in broiler chickens challenged with Salmonella Enteritidis lipopolysaccharides. Poult. Sci. 2015, 94, 2887-2897. [CrossRef] [PubMed]

33. Adhikari, P.; Cosby, D.E.; Cox, N.A.; Franca, M.S.; Williams, S.M.; Gogal, R.M.; Ritz, C.W.; Kim, W.K. Effect of dietary fructooligosaccharide supplementation on internal organs Salmonella colonization, immune response, ileal morphology, and ileal immunohistochemistry in laying hens challenged with Salmonella Enteritidis. Poult. Sci. 2018, 97, 2525-2533. [CrossRef]

34. Brito, J.R.F.; Xu, Y.; Hinton, M.; Pearson, G.R. Pathological findings in the intestinal tract and liver of chicks after exposure to Salmonella serotypes Typhimurium or Kedougou. Br. Vet. J. 1995, 151, 311-323. [CrossRef]

35. Zhang, B.; Li, G.; Shahid, M.S.; Gan, L.; Fan, H.; Lv, Z.; Yan, S.; Guo, Y. Dietary l-arginine supplementation ameliorates inflammatory response and alters gut microbiota composition in broiler chickens infected with Salmonella enterica serovar Typhimurium. Poult. Sci. 2020, 99, 1862-1874. [CrossRef] [PubMed]

36. Van Immerseel, F.; De Buck, J.; De Smet, I.; Mast, J.; Haesebrouck, F.; Ducatelle, R. Dynamics of immune cell infiltration in the caecal lamina propria of chickens after neonatal infection with a Salmonella Enteritidis strain. Dev. Comp. Immunol. 2002, 26, 355-364. [CrossRef]

37. Wigley, P. Immunity to bacterial infection in the chicken. Dev. Comp. Immunol. 2013, 41, 413-417. [CrossRef]

38. Xie, S.; Li, Y.; Zhao, S.; Lv, Y.; Yu, Q. Salmonella infection induced intestinal crypt hyperplasia through Wnt/ß-catenin pathway in chicken. Res. Vet. Sci. 2020, 130, 179-183. [CrossRef]

39. Shao, Y.; Guo, Y.; Wang, Z. $\beta-1,3 / 1,6$-Glucan alleviated intestinal mucosal barrier impairment of broiler chickens challenged with Salmonella enterica serovar Typhimurium. Poult. Sci. 2013, 92, 1764-1773. [CrossRef]

40. Shao, Y.; Lei, Z.; Yuan, J.; Yang, Y.; Guo, Y.; Zhang, B. Effect of zinc on growth performance, gut morphometry, and cecal microbial community in broilers challenged with Salmonella enterica serovar Typhimurium. J. Microbiol. 2014, 52, 1002-1011. [CrossRef]

41. Almeida, J.A.S.; Ponnuraj, N.P.; Lee, J.J.; Utterback, P.; Gaskins, H.R.; Dilger, R.N.; Pettigrew, J.E. Effects of dietary clays on performance and intestinal mucus barrier of broiler chicks challenged with Salmonella enterica serovar Typhimurium and on goblet cell function in vitro. Poult. Sci. 2014, 93, 839-847. [CrossRef]

42. Rajani, J.; Dastar, B.; Samadi, F.; Karimi-Torshizi, M.A.; Abdulkhani, A.; Esfandyarpour, S. Effect of extracted galactoglucomannan oligosaccharides from pine wood (Pinus brutia) on Salmonella Typhimurium colonisation, growth performance and intestinal morphology in broiler chicks. Br. Poult. Sci. 2016, 57, 682-692. [CrossRef]

43. Aljumaah, M.R.; Alkhulaifi, M.M.; Abudabos, A.M.; Alabdullatifb, A.; El-Mubarak, A.H.; Al Suliman, A.R.; Stanley, D. Organic acid blend supplementation increases butyrate and acetate production in Salmonella enterica serovar Typhimurium challenged broilers. PLOS ONE 2020, 15, e0232831. [CrossRef]

44. Liu, J.D.; Bayir, H.O.; Cosby, D.E.; Cox, N.A.; Williams, S.M.; Fowler, J. Evaluation of encapsulated sodium butyrate on growth performance, energy digestibility, gut development, and Salmonella colonization in broilers. Poult. Sci. 2017, 96, 3638-3644. [CrossRef] 
45. Kelly, C.; Gundogdu, O.; Pircalabioru, G.; Cean, A.; Scates, P.; Linton, M.; Pinkerton, L.; Magowan, E.; Stef, L.; Simiz, E.; et al. The in vitro and in vivo effect of carvacrol in preventing Campylobacter infection, colonization and in improving productivity of chicken broilers. Foodborne Pathog. Dis. 2017, 14, 341-349. [CrossRef] [PubMed]

46. Ijaz, U.Z.; Sivaloganathan, L.; McKenna, A.; Richmond, A.; Kelly, C.; Linton, M.; Stratakos, A.C.; Lavery, U.; Elmi, A.; Wren, B.W.; et al. Comprehensive longitudinal microbiome analysis of the chicken cecum reveals a shift from competitive to environmental drivers and a window of opportunity for Campylobacter. Front. Microbiol. 2018, 9, 2452. [CrossRef] [PubMed]

47. Richards, P.; Fothergill, J.; Bernardeau, M.; Wigley, P. Development of the caecal microbiota in three broiler breeds. Front. Vet. Sci. 2019, 6, 201. [CrossRef] [PubMed]

48. Kempf, F.; Menanteau, P.; Rychlik, I.; Kubasová, T.; Trotereau, J.; Virlogeux-Payant, I.; Schaeffer, S.; Schouler, C.; Drumo, R.; Guitton, E.; et al. Gut microbiota composition before infection determines the Salmonella superand low-shedder phenotypes in chicken. Microb. Biotechnol. 2020, 13, 1611-1630. [CrossRef] [PubMed]

49. Rychlik, I. Composition and function of chicken gut microbiota. Animals 2020, 10, 103. [CrossRef]

Publisher's Note: MDPI stays neutral with regard to jurisdictional claims in published maps and institutional affiliations.

(C) 2020 by the authors. Licensee MDPI, Basel, Switzerland. This article is an open access article distributed under the terms and conditions of the Creative Commons Attribution (CC BY) license (http://creativecommons.org/licenses/by/4.0/). 